\title{
Antibodies against heparin-protamine complexes: Effect and clinical relevance
}

\author{
Ziv Beckerman, MD, and Oved Cohen, MD
}

From the Department of Cardiac Surgery, Rambam Health Care Campus, Haifa, Israel.

Disclosures: Authors have nothing to disclose with regard to commercial support.

Received for publication Aug 2, 2015; accepted for publication Aug 3, 2015; available ahead of print Aug 28, 2015 .

Address for reprints: Ziv Beckerman, MD, Department of Cardiac Surgery, Rambam Health Care Campus, PO Box 9602, Haifa 31096, Israel (E-mail: z_beckerman@rambam.health.gov.il).

J Thorac Cardiovasc Surg 2015;150:974-5

$0022-5223 / \$ 36.00$

Copyright (c) 2015 by The American Association for Thoracic Surgery

http://dx.doi.org/10.1016/j.jtcvs.2015.08.003

The study by Grieshaber and colleagues, "Plateletactivating protamine-heparin-antibodies lead to higher protamine demand in patients undergoing cardiac surgery," in this issue of the Journal, ${ }^{1}$ is a prospective study that joins other recent publications aiming to answer an intriguing clinical question: Do antibodies against heparin-protamine complexes have a significant clinical influence?

Protamine is associated with various postoperative side effects. It is a strongly basic substance that combines with the strongly acidic heparin to form a stable (salt) conjugated pair that has no anticoagulant activity. The interaction of protamine with heparin leads to further dissociation of the heparin-antithrombin III complex, resulting in loss of its anticoagulant activity.

Antibodies and immunologic reactions against protamine have been widely demonstrated. Additionally, it has been demonstrated that patients develop antibodies against protamine-heparin complexes after cardiac surgery. Such an immune reaction resembles that of heparin and platelet factor 4, which is well known to induce the causative antibodies of heparin-induced thrombocytopenia (HIT).

The prevalence of antibodies against these complexes in patients undergoing cardiac surgery ranges between $1 \%$ and $9.6 \%$ preoperatively increasing to $26.6 \%$ to $31 \% 10$ and 30 days postoperatively.

The authors attempted to analyze the perioperative prevalence of platelet-activating antibodies against protamineheparin complexes (PrAB) in patients undergoing cardiac surgery on cardiopulmonary bypass (CPB) and their influence on clinical outcome.

The study by Grieshaber and colleagues was an observational, prospective single-center study that aimed to describe the prevalence and time-course of occurrence of PrAB.

Blood samples were obtained 1 day before operation (T0), 1 hour after weaning from CPB (T1), as well as 24 hours (T2), and 7 days (T3) after operation.

All serologic analyses were conducted in a blinded fashion after completion of the inclusion period. Testing was done using a modified heparin-induced platelet activation assay protocol.

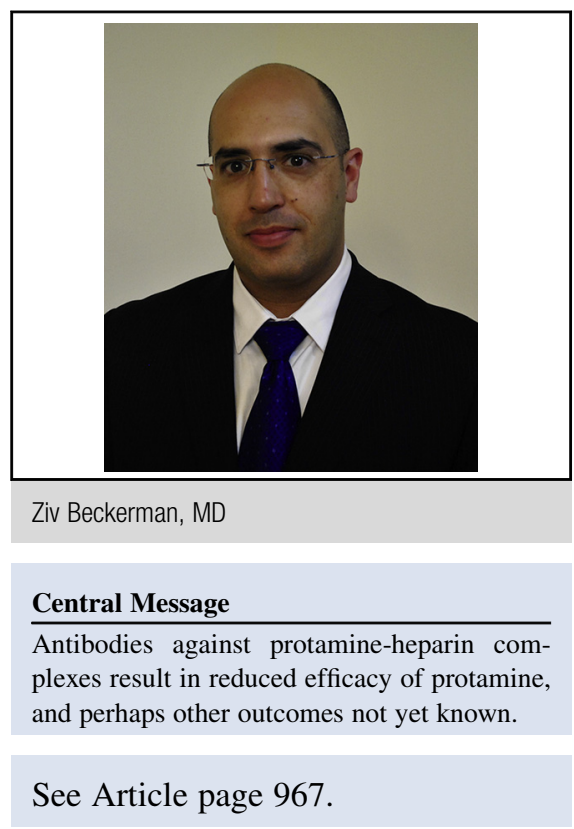

The authors enrolled 200 patients, with only 185 patients included in the final diagnoses (15 patients [7.5\%] were excluded due to loss of follow-up). Of the 185 patients analyzed, $24(13.0 \%)$ were positive for PrAB at T0. These patients differed from the preoperative negative group only in their age (being significantly older). At T1 and T2, PrAB were detected in $13.6 \%$ (21 out of 155 patients) and $14.2 \%$ (22 out of 155 patients), respectively. Only 155 patients were included in these analyses because in 30 patients the antibody status could not be established. At T3, reactivity was present in $11.9 \%$ (22 out of 185 patients). Altogether, 42 patients $(22.7 \%)$ showed reactivity at any time point.

Patients positive for $\operatorname{PrAB}$ at baseline were found to receive higher doses of protamine.

The authors further established the HIT antibody status of the patients to exclude potential effect of these antibodies. Concurrently the groups were divided to 4: group 1 ( $\mathrm{n}=97$ ) was patients negative for both PrAB and HIT antibodies, group $2(\mathrm{n}=39)$ was patients positive for PrAB and negative for HIT antibodies, group $3(n=19)$ was patients negative for $\operatorname{PrAB}$ and positive for HIT antibodies, and group $4(n=30)$ was patients with unknown antibody status at any time point.

No patient was positive for HIT antibodies at baseline. No patient was positive for both PrAB and HIT antibodies at any time point. No significant differences in platelet count variability were noted postoperatively. 
Patients positive for PrAB at any time point had no higher probability to receive red blood cells or platelet transfusions perioperatively. The total drainage volumes from surgical drains were comparable in both groups, as was the prevalence of reexploration. A combined major cardiovascular and cerebrovascular events end point did not significantly differ between the groups.

Taken together, the major clinical finding demonstrate that preoperative presence of $\operatorname{PrAB}$ is associated with increased protamine demand for heparin neutralization after CPB. As the authors state, the increased use of protamine in PrAB-positive patients might be attributed to a reduced protamine effect following PrAB binding to protamine.

Clinical evidence from this and other studies support the belief that platelet-activating antibodies against protamine-heparin complexes have biologic consequences. These consequences clearly include a reduced efficacy of protamine and perhaps other outcomes not known yet.

The major strength of this work is its prospective, blinded nature. Nevertheless, the small number of patients included in this study is a limitation disabling a final conclusion on clinical effects, especially with regard to thromboembolic events.

The authors state that they do not currently propose a standard preoperative screening for presence of PrAB. I only partially agree with that statement. It is my opinion that until stronger evidence is demonstrated it can be useful to study the effects of $\operatorname{PrAB}$ in all patients. Such information will assist in future characterization of the possible clinical effects of these antibodies.

Additionally, in our evolving era of multiple cardiac surgeries and as part of the decision-making process before redo surgery versus possible minimally invasive procedures, the effects of such antibodies might be relevant. A study on the effects of such antibodies in redo cases might be of interest.

\section{Reference}

1. Grieshaber P, Bakchoul T, Wilhelm J, Wagner A, Wollbrück M, Böning A Sachs U. Platelet-activating protamine-heparin-antibodies lead to higher protamine demand in patients undergoing cardiac surgery. J Thorac Cardiovasc Surg. 2015;150:967-73.e1. 